\title{
Proximal Gastrectomy with Anti-reflux Anastomosis for Patients with Adenocarcinoma of Esophagogastric Junction: A Simple and Safe Triangle-Valve Technique
}

\section{Yongshun Gao ( $\nabla$ gaoys@zzu.edu.cn )}

Zhengzhou University First Affiliated Hospital

Jiangang Sun

Zhengzhou University First Affiliated Hospital

\section{Yuheng Chen}

Chinese Academy of Medical Sciences and Peking Union Medical College

\section{Yunfei Zhang}

Zhengzhou University First Affiliated Hospital

\section{Peng Chen}

Zhengzhou University First Affiliated Hospital

\section{Liang Zong}

Yangzhou University

\section{Jingjing Huang}

Zhengzhou University First Affiliated Hospital

\section{Ji Han}

Zhengzhou University First Affiliated Hospital

\section{Xiaoping Chen}

Tongji Hospital of Tongji Medical College of Huazhong University of Science and Technology

\section{Technical innovations}

Keywords: Proximal Gastrectomy, Anti-reflux Anastomosis, Adenocarcinoma of Esophagogastric Junction

Posted Date: February 13th, 2020

DOI: https://doi.org/10.21203/rs.2.23460/v1

License: (9) This work is licensed under a Creative Commons Attribution 4.0 International License. Read Full License 
Version of Record: A version of this preprint was published at Molecular and Clinical Oncology on September 7th, 2020. See the published version at https://doi.org/10.3892/mco.2020.2132. 


\section{Abstract}

Background A simple and safe triangle-valve technique (TVT) was applied to proximal gastrectomy (PG) in order to prevent post-surgery gastric reflux among patients with adenocarcinoma of the esophagogastric junction (AEG). Its clinical outcomes were evaluated in comparison to those of the canonical total gastrectomy (TG).

Method This retrospective study of 74 AEG patients compared two surgical procedures PG-TVT $(n=44)$ and TG $(n=30)$ in terms of their surgical outcomes, postoperative complications, and nutritional status. All patients were followed-up for 6 months. Reflux Disease Questionnaire (RDQ) was used to evaluate reflux esophagitis. patients with the RDQ score of $\geq 12$ points were diagnosed with gastroesophageal reflux disease (GERD).

Results The mean operation time was significantly shorter in PG-TVT group (242.6 minutes) than in TG group (288.1 minutes). The overall postoperative complication rate was not significantly different between PG-TVT and TG groups. All patients were followed up by 6 months. None of them developed cancer recurrence in distant organs, remnant stomach, or lymph nodes. The GERD incidence was similar between PG-TVT and TG groups. The mean levels of total protein and albumin within the 6 months were significantly higher in PG-TVT group than in TG group after adjusting the time effect and the interaction of time and surgical methods. The level of total protein significantly increased within 6 months in PG-TVT but decreased in TG group.

Conclusion PG-TVT provides several advantages including a shorter operating time, better postoperative nutritional status, and similar incidence of GERD over TG for AEG patients.

\section{Background}

Adenocarcinoma of the esophagogastric junction (AEG), distinct from noncardiac gastric cancer, is defined as a type of cancer centered within $5 \mathrm{~cm}$ proximal and distal of the anatomical cardia[1]. In recent decades, there has been a dramatic increase in the incidence of AEG not only in Western countries[2, 3] but also in Asian countries[4-6]. In China, a significantly increased trend of AEG was reported from 1988 to 2013 in a population-based study, while the incidence of noncardiac gastric cancer dropped[7].

Although an AEG classification system, including types I to III, has been proposed by Siewert and Stein to aid clinicians in the decision of surgical approach[8], surgical strategies for AEG cases remain controversial. Total gastrectomy (TG) is considered as a standard procedure with benefits of enough resection margin and more radical lymphadenectomy[9]. Some recent studies have reported that proximal gastrectomy $(P G)$ achieves survival rates equivalent to those of TG while preserving the physiologic functions of the gastric remnant[10-14]. However, others suspect whether the advantages of PG overweight the functional drawbacks of esophageal reflux, which substantially affects quality of life[15, 16], since several reconstruction methods after PG, such as esophagogastrostomy, and jejunal interposition, might carry a high risk of reflux esophagitis and gastroesophageal anastomotic 
stenosis[16-20]. Recently, Hayami et al. applied a novel double-flap technique, invented by Kamikawa et al.[21], to laparoscopic proximal gastrectomy (LPG-DFT) to prevent reflux. Their results showed LPG-DFT is a better surgical procedure for upper-third early gastric cancer than laparoscopic total gastrectomy in terms of morbidity, postoperative hospital stay, and postoperative nutritional status[22]. However, the operation time was significantly longer in LPG-DFT group due to the complexity of valvuloplasty, which demands masterful intracorporeal suturing.

Here, we introduce a simple and safe anti-reflux anastomosis, named "Triangle-Valve Technique" (TVT), to proximal gastrectomy (PG-TVT). This valve technique was designed to prevent anti-reflux. We hypothesize that the TVT would be a time-saving technique due to its easiness and simplicity; the clinical outcomes of PG-TVT would be the same as TG's in terms of postoperative complication; PG-TVT might improve their nutritional status by conserving half of stomach.

\section{Patients And Methods}

\section{Patients}

74 patients with AEG (Siewert II or III) were recruited consecutively at First Affiliated Hospital of Zhengzhou University from July 2013 to December 2017. From May 2015 to December 2017, PG-TVT was performed on 44 patients with clinical diagnosis of $\mathrm{T}_{1-4} \mathrm{~N}_{0-3} \mathrm{M}_{0}$ at the preoperative evaluation according to the 8th edition of American Joint Committee on Cancer Tumor-node-metastasis (AJCCTNM) Staging System of Gastric Carcinoma. 30 patients with $\mathrm{T}_{1-4} \mathrm{~N}_{0-3} \mathrm{M}_{0}$ located in or involving the upper third of the stomach received TG from July 2013 to December 2015 as the control group. From January 2016 to 2017, TG was also performed on another 17 patients with the same indications as in the previous period. However, the latter period was not selected because the number of TG-TVT cases had gradually increased during that period. Certain settings, including the surgeon's preference in relation to adopting the procedure, had been taken into considerations to avoid selection bias. All patients were operated by the same surgical team.

All patients were in stable and operable condition at operation. Written informed consent was obtained from all of them. This study is a retrospective study using clinicopathological, surgical, and follow-up data, and has approved by the institutional review board at the First Affiliated Hospital of Zhengzhou University, Zhengzhou, China.

\section{Surgical Procedure of PG and PG-TVT Reconstruction}

The detailed surgical procedure of PG and PG-TVT reconstruction is described as below (Figure 1). After lymph node dissection is completed, the first step of procedure is to locate tumor lesion and determine resection region including upper (G1) and lower (G2) resection margins. As shown in Figure 1A, an example of a primary tumor lesion located along lesser curvature (shown in black) is used to present the resection region with upper $\mathrm{G} 1$ and lower $\mathrm{G} 2$ margins at distance of no less than $2 \mathrm{~cm}$ and $5 \mathrm{~cm}$ respectively from tumor lesion. The before-mentioned distances are measured on tension-free gastric 
body. Based on the after-mentioned estimation on resection region, the upper resection line is defined with cutting line G1 (black), and then a lower resection line is defined with the first cutting line L1 (red line) which is perpendicular to greater curvature and second cutting line L2 (blue line) which is at 30 degrees relative to line L1 (Figure 1B). The crossing point of line L2 on lesser curvature is supposed to be no less than $8 \mathrm{~cm}$ from pyloric sphincter. Consequently, a solid linear path of L1 and L2 as shown in figure 1B indicates the lower resection line. A proximal gastrectomy was performed along the above solid linear path with a linear cutting closure (Figure 1B). Thirdly, as shown in figure 1C, on the exposed flattened side of the remnant gastric body, a curve line (blue) is drawn $2 \mathrm{~cm}$ from greater curvature with 3 parallel lines (red) $(2 \mathrm{~cm}$ from each other) perpendicular to it. The midpoints of those three parallel lines are marked as shown in Figure 1C. Similar curve line, other three parallel lines and mid-points are also drawn on the back side of the remnant gastric body (not shown in Figure 1C). Then, three stitches are done along those midpoints on both sides (Figure 1D) so that the gastric wall between those midpoints of both sides will be folded towards gastric cavity to form a Triangle-Valve shaped bulge when those sutures are knotted (Figure 1E). Finally, the distal remnant stomach is anastomosed to the esophageal end through point $\mathrm{H}$ (Figure 1E). The triangle valve shaped bulge as shown in Figure $1 \mathrm{~F}$ functions similarly to cardia as an anti-reflux design.

\section{Surgical Procedure of TG and Roux-en-Y (R-Y) Reconstruction}

TG radical resection was performed by following Japanese gastric cancer treatment guidelines [23]. The resection distance from the upper and lower margins of tumor must be $\geq 2 \mathrm{~cm}$, and D2 lymph node dissection must be ensured [24, 25]. After that, Roux-en-Y reconstruction was completed [26]. Jejunum was separated $20 \mathrm{~cm}$ below the ligament of Treitz and esophageal distal jejunal anastomosis was performed. Then proximal jejunum was anastomosed with the distal jejunum $40 \mathrm{~cm}$ below the anastomosis.

\section{Clinical Parameters and Surgical Outcomes}

Patients' clinical features were obtained from their medical records: age, sex, body mass index (BMI), Siewert type, tumor size, histological type, pathological TNM stage, history of abdominal surgery, preoperative chemotherapy, and postoperative adjuvant chemotherapy. Surgical findings such as operation time, estimated blood loss, combined with laparoscopic-assisted, extent of lymph node dissection, number of retrieved lymph nodes, residual tumor (R), postoperative complications, and postoperative hospital stay were retrieved from their records as well.

\section{Follow-up and Postoperative Nutritional Status}

All patients were followed up for 6 months. Reflux Disease Questionnaire (RDQ) was used to evaluate reflux esophagitis. The frequency and severity of the upper gastrointestinal symptoms (heartburn, regurgitation, noncardiogenic chest pain, and regurgitation) were asked in 6 months after surgery. Patients with RDQ score of $\geq 12$ points were diagnosed with gastroesophageal reflux disease (GERD) [27]. To evaluate postoperative nutritional status, changes in body weight and biochemical data such as 
serum concentrations of total protein (TP), albumin (Alb), hemoglobin $(\mathrm{Hb})$, prealbumin (PA) were examined at 7days, and 6 months after surgery.

\section{Statistical analysis}

Categorical variables were compared by using chi-square test or Fisher's Exact Test, and continuous data were compared by using Student's t-test or Mann-Whitney Utest. Postoperative changes in weight, TP, Alb, $\mathrm{Hb}$ and PA were compared using repeated measures ANOVA and Post-Hoc Tests for the multiple comparisons. All analysis was conducted using RStudio software (Version 1.1.456, 2009-2018 RStudio Inc.). All Statistical tests were two-sided, and $p<0.05$ was considered statistically significant.

\section{Results}

\section{Patient Characteristics}

The characteristics of patients are shown in Table 1. There were no significant differences in age, sex, $\mathrm{BMI}$, previous abdominal operation, Siewert type, histological type, pathological TNM stage, preoperative and postoperative chemotherapy between two groups. The median tumor size was significantly larger for patients with TG $(4.6 \mathrm{~cm})$ than for those with PG-TVT $(3.5 \mathrm{~cm})$.

\section{Surgical outcome}

Table 2 shows operative and early postoperative outcomes of patients undergoing PG-TVT and TG respectively. The mean operation time was significantly shorter in PG-TVT group (242.6 minutes) than in TG group (288.1 minutes). There was no significant difference in the estimated blood loss, transfusion, laparoscopic assisted, extend of lymph node dissection, and the number of retrieved lymph nodes between two groups. R0 resection was performed in all patients and no mortality was recorded across all patients. The overall postoperative complication rate was not significantly different between PG-TVT and TG groups $(22.7 \%$ vs. $20.0 \%, p=1.000)$, including the frequency of anastomotic complications, infection, and lymphatic fistula. The mean postoperative hospital stay of patients was shorter in PG-TVT group (16 days) than in TG group (17 days), but difference was not statistically significant.

\section{Follow-up and Postoperative Nutritional Status}

All patients were followed up for 6 months. None of them developed cancer recurrence in distant organs, remnant stomach, or lymph nodes. For the incidence of GERD within 6 months after PG-TVT and TG, GERD was observed in 7 of 44 PG-TVT patients (15.9\%), compared to 4 of 30 patients in TG group (13.3\%), but the difference was not significant $(p=1.00)$.

The mean weight loss of patients in 6 months was $1.9 \mathrm{~kg}$ in TG group and $2.0 \mathrm{~kg}$ in PG-TVT group but there was no significant difference ( $p=0.743$, data not shown). Figure 2 shows the means of $\mathrm{Hb}$, Alb, TP and PA at 3 timepoints (pre-operation, 1 week and 6 months after surgeries) in PG-TVT and TG respectively. Adjusting the time effect and interaction of time and surgical methods, the mean levels of 
TP and Alb in 6 months were significantly higher in PG-TVT than in TG group. Also, the level of TP was significantly increased at 6 months in PG-TVT group but decreased in TG group. The mean levels of $\mathrm{Hb}$ and PA at 6 months were also higher in PG-TVT group than in TG group, but not significantly. As shown in Figure 2, levels of all biomarkers dropped after the surgery but have been increasing in the 6 months at PG-TVT group. However, levels of all biomarkers, except Alb, decreased at 6 months at TG group. The detailed information is shown in supplemental table 1.

\section{Discussion}

In this study, operating time for PG-TVT was much shorter than that for TG. The nutritional status of patients in PG-TVT group showed a significant advantage over those in TG group. There were no significant differences between the two groups in the frequencies of complications including reflux esophagitis, and the postoperative weight loss in 6 months. Thus, PG-TVT provided good clinical results to patients with AEG.

Proximal gastrectomy used to treat AEG patients is controversial. Important considerations include curability and prognosis related to the surgical treatment, as well as the development of complications and postoperative life quality. It was widely believed that proximal gastrectomy reduces postoperative weight loss [11] due to conserving half of the stomach and achieves survival rates equivalent to those of total gastrectomy [28]. However, several studies reported proximal gastrectomy was associated with a markedly higher complication rate and the functional drawbacks of esophageal reflux, which substantially affects quality of life, compared to the total gastrectomy [16-20]. In our study, the postoperative morbidity of PG-TVT had no difference compared to that of TG group.

In many cases, the most common problem after PG is reflux. Kim et al reported the rate of reflux esophagitis at $48 \%$ after proximal gastrectomy[29] and Katsoulis et al reported $100 \%$ of patients experienced reflux symptoms after proximal gastrectomy[15]. In the recent study, Hayami et al. reported no severe reflux esophagitis observed after the novel double-flap technique, LPG-DFT. In LPG-DFT procedure, valvuloplasty preserves the backflow prevention valve embedded between the submucosal layer and the seromuscular flap of the stomach. However, due to the complex valvuloplasty, it demands masterful intracorporeal suturing and the operating time was much longer than that of the total gastrectomy.

In our study, TVT was applied in proximal gastrectomy, no patients developed reflux esophagitis in PGTVT group in 6 months follow-up, compared to 3 patients at TG group. TVT was designed for anti-reflux principle. Stomach wall was sewed from the small side of the stomach into the stomach. When the contraction of the stomach was flat, it was a triangle valve, like the cardia. It was difficult to reflux gastric juice due to the narrow threshold. When the stomach diastoles, there was a peak hillock, which was the key to anti-reflux. When the gastric acid or gastric fluid impacted upward, according to the physical principle, the collision peak would generate vortex phenomenon, which will greatly reduce the reflux situation and gastric juice is also difficult to harm the relatively fragile anastomosis. The procedure of 
TVT is easy and simple and does not demand masterful suturing, therefore the operating time for PG-TVT was much shorter than that for TG.

In terms of the nutritional status, the postoperative levels of TP and Alb were significantly higher in PGTVT than in TG group. No significant difference of $\mathrm{Hb}$ and PA was observed between the two groups. However, the Hb level increased slightly within 6 months in PG-TVT group but decreased in TG group. It could benefit from the present of the gastric remnant. Levels of several hormones such as ghrelin and gastrin are decreased after gastrectomy [30,31]. However, there is less loss of serum vitamin B12 level and hormones at the group of PG-TVT. In addition, the appetite increased due to the lower incidence of GERD. These results indicate that compared with TG, PG-TVT showed a significant advantage.

Although the weight loss and other biomarkers did not show significant difference between the two groups, there was a positive trend observed at PG-TVT group. The results are consistent with those from other studies [22]. The patients were only followed up for 6 months in our study. Thus, the long-time follow-up is needed.

The present study has some limitations. First, this is a retrospective study with a small sample size and was conducted at a single institution. However, the two operative procedures were performed with the same surgeons at the same institution. Clinicopathologic and treatment factors, Siewert type, degree of lymph node dissection, and the degree of lymph node involvement were similar between the two groups studied. Thus, the bias from patients and surgeons were minimized. Second, a randomized clinical trial with equivalent background characteristics among the reconstructions after PG is required to further analyze the advantages of PG-TVT. Another limitation is the relatively short follow-up time. The long follow-up period and more nutritional indexes should be included in the future analysis.

\section{Conclusion}

In conclusion, PG-TVT provides several advantages including a shorter operating time, better postoperative nutritional status, and similar incidence of GERD over TG for AEG patients. Further randomized clinical trials with a large sample size will be required to fully investigate the comparative benefits of PG-TVT, and further evaluation of patients' quality of life and survival analysis compared with that after traditional total gastrectomy are warranted.

\section{Declarations}

\section{Acknowledgements}

Not applicable.

\section{Authors' contributions}


Yongshun Gao was responsible for conception and design, analysis and interpretation of data and preparation of the manuscript. Jiangang Sun, Yuheng Chen, Yunfei Zhang, Peng Chen, Ji Han and Jingjing Huang were responsible for the acquisition of data and interpretation of data. Liang Zong and Xiaoping Chen were responsible for conception and design, revision and final approval of the manuscript. All authors read and approved the final manuscript.

\section{Funding}

The fund for this study was provided by the Wu Jieping Medical Foundation; grant no. 320.2710.1847.

\section{Availability of data and materials}

The datasets generated and/or analysed during the current study are available from the corresponding author on request.

\section{Ethics approval}

The study with human samples was approved by the Ethics Committee of the First Affiliated of Zhengzhou University. And we clarify that all clinical samples described here were gained from patients who had given written informed consent.

\section{Consent for publication}

Not applicable as there were no identifiable materials included in this manuscript.

\section{Competing interests}

The authors declare that they have no competing interests.

\section{References}

1. Brierley J, Gospodarowicz MK, Wittekind C, TNM classification of malignant tumours (2017) Wiley Blackwell, Oxford

2. Buas MF, Vaughan TL (2013) Epidemiology and risk factors for gastroesophageal junction tumors: understanding the rising incidence of this disease. Seminars in radiation oncology 23:3-9

3. Devesa SS, Blot WJ, Fraumeni JF, Jr. (1998) Changing patterns in the incidence of esophageal and gastric carcinoma in the United States. Cancer 83:2049-2053

4. Kim JJ (2018) Epidemiology of Gastroesophageal Junction Adenocarcinoma in Korea. Journal of gastric cancer 18:328-338

5. Blaser MJ, Saito D (2002) Trends in reported adenocarcinomas of the oesophagus and gastric cardia in Japan. European journal of gastroenterology \& hepatology 14:107-113

6. Zhou Y, Zhang Z, Zhang Z, et al (2008) A rising trend of gastric cardia cancer in Gansu Province of China. Cancer letters 269:18-25 
7. Liang D, Liang S, Jin J, et al (2017) Gastric cancer burden of last 40 years in North China (Hebei Province): A population-based study. Medicine 96:e5887

8. Siewert JR, Stein HJ (1998) Classification of adenocarcinoma of the oesophagogastric junction. The British journal of surgery 85:1457-1459

9. Isguder AS, Nazli O, Tansug T, et al (2005) Total gastrectomy for gastric carcinoma. Hepatogastroenterology 52:302-304

10. Harrison LE, Karpeh MS, Brennan MF (1998) Total gastrectomy is not necessary for proximal gastric cancer. Surgery 123:127-130

11. Katai H, Sano T, Fukagawa T, et al (2003) Prospective study of proximal gastrectomy for early gastric cancer in the upper third of the stomach. The British journal of surgery 90:850-853

12. Shiraishi N, Adachi Y, Kitano S, et al (2002) Clinical outcome of proximal versus total gastrectomy for proximal gastric cancer. World journal of surgery 26:1150-1154

13. Solerio D, Camandona M, Gasparri G, et al (2003) [Adenocarcinoma of the cardia: surgical strategies compared]. Tumori 89:143-148

14. Aitaliev MS, Zemlianoi VP, Nepomniashchaia SL (2005) [Evaluation of surgical traumaticity of standard and extended surgical procedures in cancer of a proximal part of the stomach]. Khirurgiia:23-26

15. Katsoulis IE, Robotis JF, Kouraklis G, et al (2006) What is the difference between proximal and total gastrectomy regarding postoperative bile reflux into the oesophagus? Digestive surgery 23:325-330

16. An JY, Youn HG, Choi MG, et al (2008) The difficult choice between total and proximal gastrectomy in proximal early gastric cancer. American journal of surgery 196:587-591

17. Ronellenfitsch U, Najmeh S, Andalib A, et al (2015) Functional outcomes and quality of life after proximal gastrectomy with esophagogastrostomy using a narrow gastric conduit. Annals of surgical oncology 22:772-779

18. Tokunaga M, Ohyama S, Hiki N, et al (2008) Endoscopic evaluation of reflux esophagitis after proximal gastrectomy: comparison between esophagogastric anastomosis and jejunal interposition. World journal of surgery 32:1473-1477

19. Wen L, Chen XZ, Wu B, et al (2012) Total vs. proximal gastrectomy for proximal gastric cancer: a systematic review and meta-analysis. Hepato-gastroenterology 59:633-640

20. Hsu CP, Chen CY, Hsieh YH, et al (1997) Esophageal reflux after total or proximal gastrectomy in patients with adenocarcinoma of the gastric cardia. The American journal of gastroenterology 92:1347-1350

21. Kamikawa Y, Kobayashi T, Kamiyama S (2001) A new procedure of esophagogastrostomy to prevent reflux following proximalgastrectomy (in Japanese). Shoukakigeka 24:1053-1060

22. Hayami M, Hiki N, Nunobe S, et al (2017) Clinical Outcomes and Evaluation of Laparoscopic Proximal Gastrectomy with Double-Flap Technique for Early Gastric Cancer in the Upper Third of the Stomach. Annals of surgical oncology 24:1635-1642 
23. Japanese Gastric Cancer A (2011) Japanese gastric cancer treatment guidelines 2010 (ver. 3). Gastric cancer : official journal of the International Gastric Cancer Association and the Japanese Gastric Cancer Association 14:113-123

24. Giacopuzzi S, Bencivenga M, Weindelmayer J, et al (2017) Western strategy for EGJ carcinoma. Gastric cancer : official journal of the International Gastric Cancer Association and the Japanese Gastric Cancer Association 20:60-68

25. Di Leo A, Zanoni A (2017) Siewert III adenocarcinoma: treatment update. Updates in surgery 69:319325

26. Aoki M, Saka M, Morita S, et al (2010) Afferent loop obstruction after distal gastrectomy with Rouxen-Y reconstruction. World journal of surgery 34:2389-2392

27. Shaw MJ, Talley NJ, Beebe TJ, et al (2001) Initial validation of a diagnostic questionnaire for gastroesophageal reflux disease. The American journal of gastroenterology 96:52-57

28. Son MW, Kim YJ, Jeong GA, et al (2014) Long-Term Outcomes of Proximal Gastrectomy versus Total Gastrectomy for Upper-Third Gastric Cancer. Journal of gastric cancer 14:246-251

29. Kim JH, Park SS, Kim J, et al (2006) Surgical outcomes for gastric cancer in the upper third of the stomach. World journal of surgery 30:1870-1876; discussion 1877-1878

30. Ariyasu H, Takaya K, Tagami T, et al (2001) Stomach is a major source of circulating ghrelin, and feeding state determines plasma ghrelin-like immunoreactivity levels in humans. The Journal of clinical endocrinology and metabolism 86:4753-4758

31. Castaneda TR, Tong J, Datta R, et al (2010) Ghrelin in the regulation of body weight and metabolism. Frontiers in neuroendocrinology 31:44-60

\section{Tables}


Table 1. Characteristics of study population

\begin{tabular}{|c|c|c|c|}
\hline Characteristics & PG-TVT $(\mathrm{N}=44)$ & $\mathrm{TG}(\mathrm{N}=30)$ & $P$ value \\
\hline Age (years) & $64(45-79)$ & $62(37-77)$ & 0.574 \\
\hline Sex (male/female) & $35 / 9$ & $28 / 2$ & 0.182 \\
\hline BMI (kg/m2) & $23.8(17.3-28.4)$ & $24.5(19.8-29.0)$ & 0.216 \\
\hline Siewert (II/III) & $27 / 17$ & $16 / 14$ & 0.655 \\
\hline \multicolumn{4}{|l|}{ Preoperative chemotherapy } \\
\hline Yes & $5(11.4)$ & $6(20.0)$ & 0.336 \\
\hline No & $39(88.6)$ & $24(80.0)$ & \\
\hline \multicolumn{4}{|l|}{ Previous abdominal operation } \\
\hline Yes & $5(11.4)$ & $6(20.0)$ & 0.336 \\
\hline No & $39(88.6)$ & $24(80.0)$ & \\
\hline Tumor size $(\mathrm{cm})$ & $3.5(0.6-10.0)$ & $4.6(1.0-10.0)$ & $<0.001$ \\
\hline \multicolumn{4}{|l|}{ T stage * } \\
\hline $\mathrm{T} 1$ & $13(29.5)$ & $3(10.0)$ & 0.095 \\
\hline T2 & $8(18.2)$ & $5(16.7)$ & \\
\hline T3 & 7 (15.9) & $3(10.0)$ & \\
\hline $\mathrm{T} 4$ & $16(36.4)$ & $19(63.3)$ & \\
\hline \multicolumn{4}{|l|}{ N stage * } \\
\hline NO & $27(61.4)$ & $20(66.6)$ & 0.507 \\
\hline N1 & $5(11.4)$ & $2(6.7)$ & \\
\hline N2 & $5(11.4)$ & $6(20.0)$ & \\
\hline N3a/N3b & $7(15.8)$ & $2(6.7)$ & \\
\hline \multicolumn{4}{|l|}{ M stage * } \\
\hline M0 & $44(100.0)$ & $30(100.0)$ & \\
\hline Proximal resection margin, $\mathrm{cm}$ & $4.6(2.5-10.0)$ & $4.9(2.0-10.0)$ & 0.796 \\
\hline Distal resection margin, $\mathrm{cm}$ & $7.4(2.5-10.5)$ & $7.0(2.0-15.0)$ & 0.093 \\
\hline \multicolumn{4}{|l|}{ Histological grading } \\
\hline Well differentiated & $6(13.6)$ & $4(13.3)$ & 1.000 \\
\hline Moderately differentiated & $21(47.7)$ & $14(46.7)$ & \\
\hline Poorly differentiated & $17(38.6)$ & $12(40.0)$ & \\
\hline \multicolumn{4}{|c|}{ Postoperative adjuvant chemotherapy } \\
\hline Yes & $28(63.6)$ & $24(80.0)$ & 0.210 \\
\hline No & $16(36.4)$ & $6(20.0)$ & \\
\hline
\end{tabular}

PG-TVT: proximal gastrectomy with triangle-valve technique; TG: total gastrectomy; BMI: body mass index.

Values are expressed as $\mathrm{n}(\%)$ or mean (range).

$P$ values were from t-test, Mann-Whitney U Test, Fisher's Exact Test, or Chi-squared test.

*: According to the 8th edition of American Joint Committee on Cancer Tumor-node-metastasis (AJCCTNM) Staging System of Gastric Carcinoma. 
able 2 Surgical outcomes of patients undergoing PG-TVT and TG

\begin{tabular}{|c|c|c|c|}
\hline Characteristics & PG-TVT $(N=44)$ & $\mathrm{TG}(\mathrm{N}=30)$ & $P$ value \\
\hline peration time (min) & $\begin{array}{r}242.6 \pm 55.3 \\
(85-355)\end{array}$ & $\begin{array}{r}288.1 \pm 58.3 \\
(210-470)\end{array}$ & 0.002 \\
\hline stimated blood loss (ml) & $\begin{array}{r}239 \pm 208 \\
(50-1200)\end{array}$ & $\begin{array}{r}297 \pm 214 \\
(100-1000)\end{array}$ & 0.100 \\
\hline \multicolumn{4}{|l|}{ ransfusion } \\
\hline Yes & $2(4.5)$ & $4(13.3)$ & 0.215 \\
\hline No & $42(95.5)$ & $26(86.7)$ & \\
\hline \multicolumn{4}{|l|}{ lith laparoscopic-assisted } \\
\hline Yes & 41 (93.2) & $24(80.0)$ & 0.146 \\
\hline No & $3(6.8)$ & $6(20.0)$ & \\
\hline \multicolumn{4}{|l|}{ ymph node dissection } \\
\hline No & 27 (61.4) & $20(66.7)$ & 0.826 \\
\hline D1+ & $17(38.6)$ & $10(33.3)$ & \\
\hline o. of retrieved lymph nodes & $32 \pm 13(9-70)$ & $36 \pm 17(18-87)$ & 0.585 \\
\hline 0 resection & $44(100)$ & $30(100)$ & \\
\hline lorbidity & $10(22.7)$ & $6(20.0)$ & 1.000 \\
\hline Anastomotic complication & $9(20.5)$ & $5(16.7)$ & \\
\hline Bleeding & $2(4.5)$ & $2(6.7)$ & \\
\hline Leakage & $3(6.8)$ & $1(3.3)$ & \\
\hline Stricture & $4(9.1)$ & $2(6.7)$ & \\
\hline Infection & $1(2.3)$ & $0(0)$ & \\
\hline Lymphatic fistula & 0 & $1(3.3)$ & \\
\hline lortality n (\%) & 0 & 0 & \\
\hline ostoperative hospital stays (day) & $16 \pm 7(8-41)$ & $17 \pm 7(8-36)$ & 0.663 \\
\hline
\end{tabular}

'G-TVT: proximal gastrectomy with triangle-valve technique; TG: total gastrectomy; BMl: ody mass index. alues are expressed as $\mathrm{n}(\%)$ or mean \pm standard deviation (range). values were from Mann-Whitney U Test, or Fisher's Exact Test.

\section{Figures}



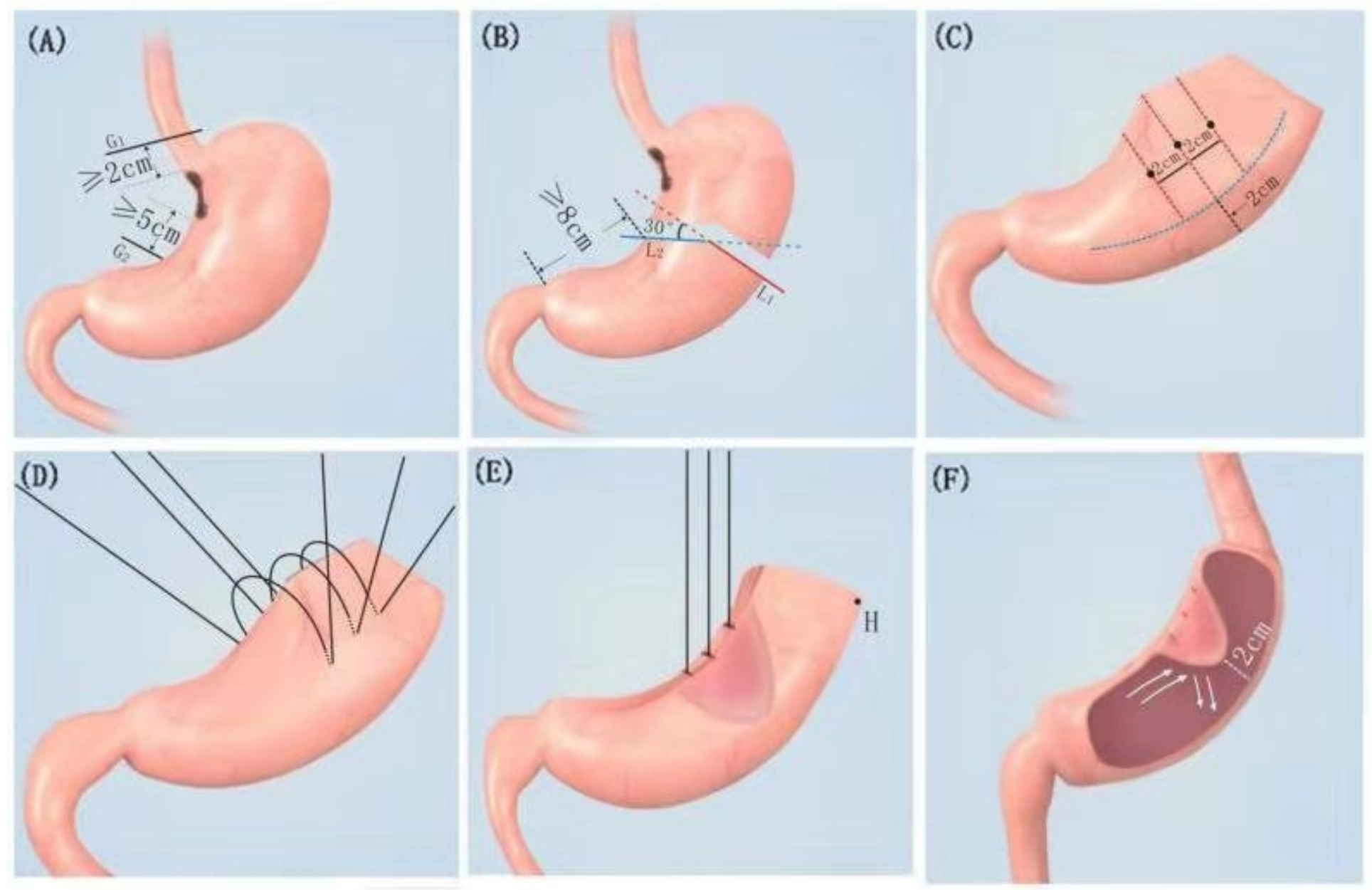

Figure 2

Schema of reconstruction with PG-TVT 

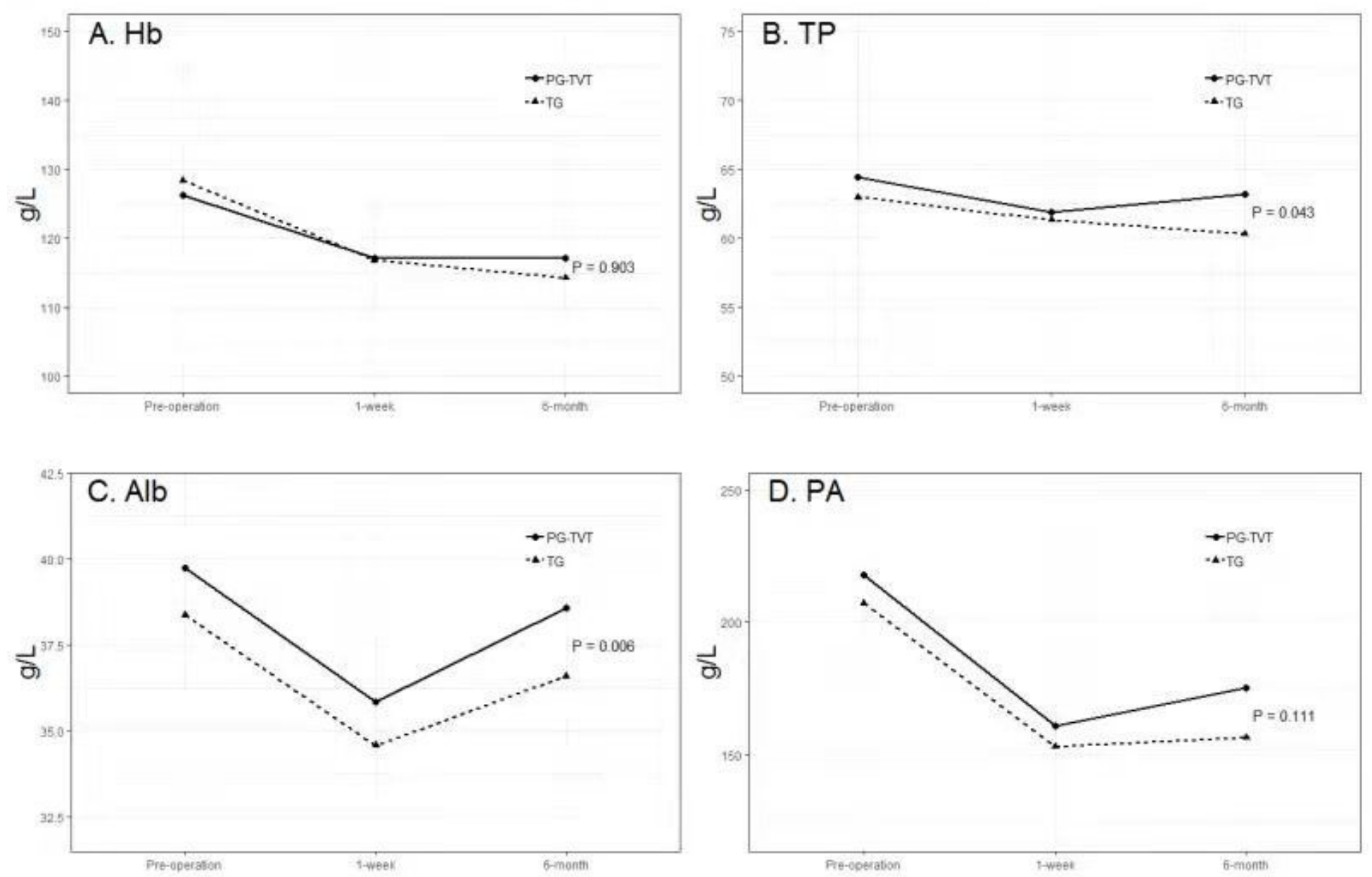

Figure 3

Comparison of changes in nutritional parameters between PG-TVT and TG. Hb hemoglobin; TP total protein; Alb albumin; PA prealbumin.

\section{Supplementary Files}

This is a list of supplementary files associated with this preprint. Click to download.

- Supplementaltable.docx

- Supplementaltable.docx 